\title{
Evaluación agronómica de nueve líneas avanzadas de arroz (Oriza sativa L.) y dos testigos comerciales bajo condiciones de riego por inundación, Sébaco, Matagalpa
}

\section{Agronomic evaluation of nine advanced rice lines (Oriza sativa L.) and two commercial varieties under flood irrigation conditions, Sébaco, Matagalpa}

\section{Víctor Aguilar Bustamante, Carlos Méndez, Esteban Treminio Artola, Lesli Loáisiga Vallecillo}

${ }^{1}$ Facultad de Agronomía, Universidad Nacional Agraria.

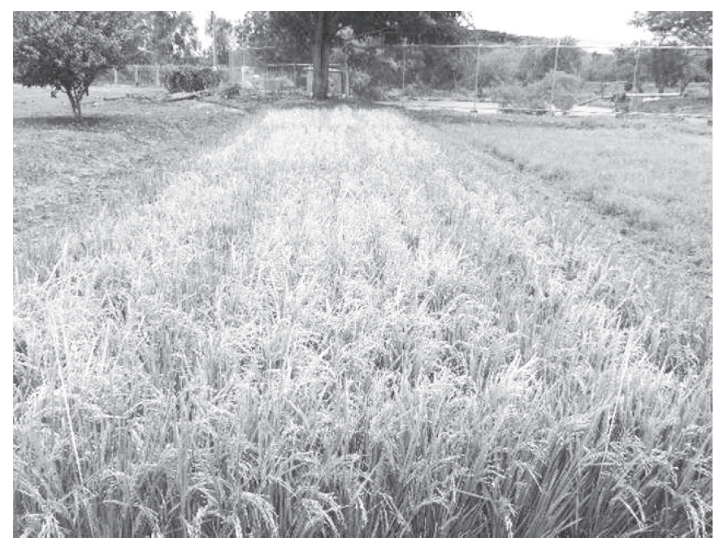

\section{RESUMEN}

El estudio se realizó en el centro experimental de arroz de Asociación Nacional de Arroceros (ANAR) en la finca Santa Rita en el Valle de Sébaco, Matagalpa con el objetivo de evaluar 9 líneas avanzadas de arroz y dos variedades con alto potencial de rendimiento y excelentes características industriales. Las parcelas experimentales fueron establecidas el 10 de Julio del 2014 y cosechadas del 12 al 14 de noviembre del mismo año. El diseño experimental fue un BCA con tres repeticiones. Los datos obtenidos fueron analizados usando ANDEVA y prueba de medias por TUKEY al 5\% de margen de error, a través del software SAS 9.1. La línea 246 y la variedad ANAR 97 presentaron cierto índice de acame y las líneas 18 y 20 presentaron una moderada longitud de excersión. Las demás líneas no presentaron acame y buena longitud de la excersión facilitando mejor la cosecha. De los 9 genotipos evaluados, 6 son altamente productivos superando a las variedades ANAR 97 y ANAR 470 oscilando sus rendimientos entre $6121 \mathrm{~kg} / \mathrm{ha}$ y $6818 \mathrm{~kg} / \mathrm{ha}$ siendo candidatos a pruebas regionales. La línea 248 presentó los mejores valores de rendimiento de arroz oro, mayor porcentaje de grano entero y mejor relación grano entero grano quebrado.

Palabras clave: arroz, crecimiento, desarrollo, rendimiento, calidad molinera

\section{ABSTRACT}

The study of nine advanced genotypes of rice and two commercial varieties was conducted at the experimental center of rice of ANAR at the Santa Rita Farm located in Sebaco, Matagalpa with the aim of assessing genotypes with higher potential yield and excellent agronomic and industrial characteristics. The experimental plots were stablished on July 10, 2014 and harvested since November 17th to November 19th of 2014. A BCA design was used with tree replications. The ANDEVA and Tukey 5\% mean comparison test was applied using SAS software 9.1. The rice plants of line 246 and the variety ANAR 97 presented certain index of felt down and the lines 18 and 20 presented a moderate length of excursion facilitating the grain of rice plants. The remainder or rice lines did not present any felt down and showed a good length excersion. From the nine studied lines, six presented the highest productivity, also higher than ANAR 97 and ANAR 470 where the yield of paddy rice found was between $6121 \mathrm{~kg} / \mathrm{ha}$ and $6818 \mathrm{~kg} / \mathrm{ha}$. These six lines can be tested in the future regional experiments. The line 248 , presented the highest yield of white rice, the higher percentage of complete rice grain and the better relationship between good grain rice and broken grain rice.

Keywords: Rice, growth, development, yield, industrial quality
Recibido: 31 de enero 2017 Aceptado: 6 de junio del 2017

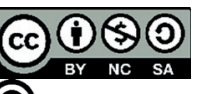

Los artículos de la revista La Calera de la Universidad Nacional Agraria, Nicaragua, se comparten bajo términos de la licencia Creative Commons: Reconocimiento, No Comercial, Compartir Igual. Las autorizaciones adicionales a las aquí delimitadas se pueden obtener en el correo freddy.aleman@ci.una.edu.ni Copyright 2017. Universidad Nacional Agraria 


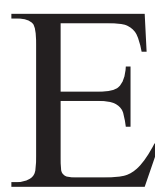
n Nicaragua el arroz (Oriza sativa L.) es un alimento básico en la dieta de la población nicaragüense, que consume actualmente $46.3 \mathrm{~kg}$ per cápita anual de arroz oro. El arroz representa el 11\% del PIB agrícola nacional. Existen actualmente unos 17,167 productores que cultivan 97,284 hectáreas, 60\% se da en condiciones de secano y un $40 \%$ en riego con mayor tecnología (INTA, 1996). Estos productores de arroz generan unos 30,000 empleos anuales.

Actualmente en Nicaragua la producción de arroz solo cubre el $80 \%$ del consumo nacional. El uso de variedades con potencial de rendimiento bajo, calidad industrial baja, uso de semilla no certificada, baja tecnología, falta de agua y suelos con problemas nutricionales son entre otros la causa por la cual no se cubre la demanda.

Es por tanto necesario ofrecer a los productores semilla mejorada con buena adaptabilidad, alto rendimiento, características agronómicas deseables y que conserve su pureza varietal. Así se lograría estabilizar los precios, la cantidad y calidad del grano, rentabilidad en la producción y se daría el gran paso a la modernización de la producción de arroz en Nicaragua.

El INTA, La Asociación Nicaragüense de Arroceros (ANAR) dedican desde hace muchos años sus mejores esfuerzos a la introducción y selección de líneas promisorias a través de pruebas preliminares de rendimiento (PPR) y las mejores pasan a pruebas avanzadas de rendimiento (PAR) para posteriormente liberarlas como variedades.

La investigación multidisciplinaria en arroz contribuye a que el sector arrocero de la región sea más eco-eficiente productivo y competitivo, así como resiliente y sostenible para que esté preparado a contribuir a la seguridad alimentaria y nutricional regional y mundial.

Debido a estas problemáticas que enfrenta el rubro arrocero, La Asociación Nicaragüense de Arroceros (ANAR), con el apoyo del Fondo Latinoamericano de Arroz de Riego (FLAR) han contribuido con fondos para la investigación y a la liberación continua de nuevas variedades con buenas características agronómicas, alto rendimiento y buena calidad industrial. Por lo que se llevó a cabo este experimento con el objetivo de evaluar las características agronómicas de nueve líneas avanzadas de arroz comparadas con dos variedades comerciales, como estrategia para la obtención de nuevas variedades.

\section{MATERIALES Y MÉTODOS}

Ubicación y descripción del área de estudio. El vivero VIOFLAR del año 2010, fue establecido en la finca Santa Rita del productor Samuel Mansell, el ensayo se estableció el 08 de Julio del 2014. La finca está ubicada en el municipio de San Isidro (Valle de Sébaco), departamento de Matagalpa, con latitud norte de 12051'07.61 y longitud Oeste de $86009^{\prime} 35.23$, con altitud de $465 \mathrm{msnm}$. La precipitación registrada fue de $390 \mathrm{~mm}$ distribuida en los meses de junio a octubre 2014 (INETER, 2015).

Sébaco, presenta una época seca de seis meses entre noviembre y abril, y una época lluviosa comprendida entre los meses de mayo y octubre. Las precipitaciones anuales se encuentran en el rango de los 738 a 850 mm/año, sin embargo, para el año 2014, las precipitaciones disminuyeron a 390 $\mathrm{mm}$ por el fenómeno del niño. La zona presenta una temperatura media anual de $26^{\circ} \mathrm{C}$, su humedad relativa promedio anual es de $73 \%$, la velocidad promedio anual de los vientos es de $3.09 \mathrm{~m} / \mathrm{s}$, con brillo solar de 7.0 horas, y evaporación anual promedio de $8.7 \mathrm{~mm}$ (INETER, 2015). Los suelos pertenecen a la serie Darío, clase II; orden vertizoles profundos, bien drenados, planos y alta fertilidad (Herrera, 2008), clasificados como zona de vida de bosque tropical seco premontano (Holdrige, 1982).

Descripción de las líneas en estudio. El Cuadro 1, muestra los genotipos de arroz evalauados en el expetimeto, su descripción y origen.

Cuadro 1. Genotipos de arroz evaluados en el Centro Experimental Santa Rita ANAR Valle de Sébaco

\begin{tabular}{lll}
\hline Tratamiento & Designación & Origen \\
\hline 18 & FL09156-4P-5-4P-2P-M & FLAR \\
71 & FL09252-2P-14-3P-1P-M & FLAR \\
72 & FL09252-2P-14-3P-2P-M & FLAR \\
73 & FL09252-2P-14-3P-3P-M & FLAR \\
78 & FL09257-5P-3-1P-1P-M & FLAR \\
90 & FL109262-3P-1-2P-1P-M & FLAR \\
109 & FL09356-3P-1-2P-2P-M & FLAR \\
246 & FL09515-7P-1-3P-2P-M & FLAR \\
248 & FL09523-5P-3-1P-1P-M & FLAR \\
ANAR 97 & ANAR 97 & Nicaragua \\
ANAR 470 & ANAR 470 & Nicaragua \\
\hline
\end{tabular}

Diseño experimental y análisis estadístico. El diseño empleado fue el de Bloques Completos al Azar (BCA), 11 tratamientos y 3 repeticiones. Cada parcela experimental fue de $15 \mathrm{~m} 2$ y cada bloque de $165 \mathrm{~m} 2$. En total el área experimental fue de $495 \mathrm{~m} 2$. Los datos se tomaron en una parcela útil de 1 m2. Se usó el programa Computarizado Statistical Analysis System (SAS) versión 9.1 (SAS INSTITUTE S INC, 2002) para realizar el análisis de varianza (ANDEVA) y la separación de medias, utilizando la prueba de rangos múltiples de Tukey $(\mathrm{p} \leq 0.05)$, para determinar su significancia.

Variables evaluadas. La medición de cada variable se realizó aplicando la escala del sistema de evaluación estándar para arroz del Centro Internacional de Agricultura Tropical (CIAT) de Colombia. Vigor: Se avaluó de manera visual en el estado fenológico 02 del ciclo vegetativo del cultivo tomando como referencia un metro cuadrado para cada tratamiento. Habilidad de macollamiento: Se registró en el estado fenológico 03 del ciclo vegetativo del cultivo, haciendo un conteo de tallos en 10 plantas tomadas al azar en el área de la parcela útil de cada genotipo. Floración: Se tomó en el estado fenológico 06. Se registró el número de días desde la emergencia hasta cuando el $50 \%$ de las plantas de la parcela estaban florecidas. Aceptabilidad fenotípica: Esta característica se evaluó en el estado fonológico 09. Acame (\%): Se tomó en el estado fenológico 09 y consistió en la 
habilidad de los tallos de permanecer erectos en el campo. Senescencia: Se evaluó de forma visual en el estado de fenológico 09. Excersión de la panícula $(\mathrm{cm})$ : Se tomó en la etapa de floración 06 al azar en 10 panículas por tratamiento y repetición de la base de la hoja de bandera hasta el nudo ciliar. Longitud de panícula $(\mathrm{cm})$ : Se tomó en el estado fenológico 09 al azar en diez panículas por tratamiento y repetición, desde el nudo ciliar hasta el último grano. Altura de Planta $(\mathrm{cm})$ : Se tomó en el estado fenológico 09 al azar en 10 plantas desde la superficie del suelo hasta la parte de la panícula más alta excluyendo la arista. Número de granos por panícula: Se midió en el estado fenológico 09. De cada parcela experimental se tomaron del área de la parcela útil 10 panículas al azar. Luego se procedió a contar el número de granos por panícula para poder obtener los promedios. Fertilidad de la panícula (\%): Se evaluó en el estado fenológico 09 y se tomaron diez panículas por parcela útil, se contaron el número de espiguillas con granos, obteniéndose así el porcentaje de fertilidad de cada tratamiento. Peso de 1000 granos (g): Se tomó en el estado fenológico 09 en tres muestras de 250 granos por línea y el promedio se multiplicó por cuatro para obtener el peso de 1000 granos con una humedad del 14\%. Rendimiento de grano en granza $(\mathrm{kg} / \mathrm{ha})$ : El rendimiento se determinó en el estado fenológico 09 en la parcela útil, arroz en granza y se expresó en $\mathrm{kg} / \mathrm{ha}$ al $24 \%$ de humedad del grano. Calidad industrial (\%): Se pesaron 200 gramos de arroz seco en granza, homogenizado y limpio con grado de humedad aproximado al $14 \%$. Se calculó el peso neto (PN) de la muestra el que se obtuvo por diferencia del peso de impurezas. Se determinó el peso de arroz integral (AI) o arroz sin cáscara. Este se sometió al pulido para obtener el peso de arroz oro (PAO). El arroz oro se clasificó en arroz entero, payana y puntilla con el uso de una criba. Por último se separó el grano yesoso de 20 gramos de arroz entero.

Manejo Agronómico del cultivo. Se realizó una limpieza general de la terraza. La preparación del suelo fue mecanizada, se realizaron dos pases con rota disco y un pase de nivelación con banqueo. La siembra fue a chorrillo (110 kg/ha) en surcos con distancia de $30 \mathrm{~cm}$ a los 12 días después de la preparación del suelo.

La fertilización se realizó a razón de $80 \mathrm{~kg}$ de nitrógeno, $52 \mathrm{~kg}$ de fósforo y $68 \mathrm{~kg}$ de potasio por hectárea. Además, se hizo una fertilización foliar con Newfol-F + Newfol-Plus + Newfol-Potásico a razón de 0.62, 0.44, y 0.62 $\mathrm{L} /$ ha más una aplicación de Newfol-Boro a razón de $0.37 \mathrm{~L} /$ ha.

A partir de los 36 dds se estableció una lámina de agua de $5 \mathrm{~cm}$, drenándose cada 8 días antes de la cosecha. Para el manejo de las malezas como la caminadora (Echinochloa colona C.) se usó Cyhalofop (Chlincher 18 EC®) a razón de $0.87 \mathrm{~L} /$ ha. Para el control de poáceas se usó Pyrazosulfuron-Ethil (Sirius $10 \mathrm{WP} \circledast$ ) a razón de $0.15 \mathrm{~kg} / \mathrm{ha} \mathrm{y}$ Metsulfuron Methyl (Ally $60 \mathrm{WG}{ }^{\circledR}$ ) a razón de $6 \mathrm{~g} /$ ha para controlar Cyperaceas y malezas de hoja ancha. Se realizaron 7 controles manuales para eliminar malezas acuáticas resistentes al control químico.
Para el control de gallina ciega (Phyllophaga sp.) y coralillo (Elasmopalpus lignosellus Z.) se utilizó Terbuphos o Counter (Quibor-Terbufos $10 \mathrm{G}($ ) a una dosis de $18.7 \mathrm{~kg}$ / ha. Para controlar las plagas del follaje y el chinche de la espiga del arroz (Oebalus insulares S.) se aplicó Deltramethrin triazophos (Rienda 21.2 EC®) a una dosis de $0.31 \mathrm{~L} / \mathrm{ha}$, Cypermetrina 35 E.C®) a una dosis de 0.25 L/ha, Clorpirifos (Pyrinex 48 EC®).

\section{RESULTADOS Y DISCUSIÓN}

Vigor. El vigor es tan importante en el cultivo de arroz para siembra directa, como para el transplante debido a que disminuye la competencia de malezas, compensa las pérdidas de las plantas (Cardoza y González, 2004). El vigor consiste en la habilidad de cubrir rápidamente los espacios entre las plantas y está influenciados por factores tales como: habilidad de macollamiento y altura de la planta entre otras CIAT (1983). Las variedades que maduran entre 110 y 140 días tienen gran vigor vegetativo (Contin, 1990). Todas las líneas estudiadas se clasificaron como genotipos muy vigorosos en su totalidad con escala 1 según escala del CIAT.

Habilidad de macollamiento (hijos por planta). Esta etapa de crecimiento es muy importante por tener una estrecha relación con el mejoramiento del cultivo y las prácticas agronómicas. En variedades tempranas el número máximo de hijos se alcanzan casi simultáneamente con la iniciación de la panícula o ligeramente después, en variedades tardías puede bien producir la elongación del tallo y la iniciación de la panícula (CIAT, 1985).

Según (Tinarelli, 1989), la intensidad e inicio del ahijamiento dependen de las características genéticas, condiciones climáticas y edáficas del lugar y técnicas agronómicas empleadas. El ANDEVA no presentó diferencia significativa entre los diferentes genotipos. En promedio ninguno de los materiales supera los 10 vástagos por planta, por lo tanto según la escala del CIAT se puede definir como mediano (10-19 hijos/planta).

Las líneas 72, 246 y la variedad ANAR 2015 presentaron mayor número de hijos con un promedio de 15 hijos por planta, contrario a estas las líneas 73 y 18 presentaron el menor número de hijos por planta con un promedio de 11 .

Floración (\%). Se inicia cuando la panícula emerge de la vaina de la hoja bandera, inmediatamente la floración es seguida por la fecundación de las flores en el tercio superior de la panícula. Entre la floración y la fecundación ocurren de 8-10 horas (Balladares y Espinoza, 1995).

La maduración del ciclo evolutivo depende no solo de las temperaturas en variedades foto sensibles, sino también de los genotipos y la interacción con otros factores ambientales. Es importante señalar que en variedades de secano se buscan variedades con periodos de floración relativamente cortos, para buscar la precocidad lo cual permitiría el escape de factores climáticos adversos como las sequías.

Vientos cálidos, secos o húmedos afectan la fecundación de los estigmas, temperaturas excesivamente bajas del agua o del aire causa esterilidad al impedir que las flores se abran y se polinicen, las panículas florecen empezando en 
la parte superior, media e inferior, esto ocurre en el primer, segundo y tercer día después de la proyección de la panícula (Espigamiento) en un ambiente tropical (Munguía y Obregón, 2000).

En general no hubo diferencia significativa entre los 11 genotipos estudiados. La mayor parte florecieron entre los 80 y 82 días a diferencia de los genotipos 90, 109, 248 y ANAR 97 que florecieron entre los 71 y 75 días.

Aceptabilidad fenotípica. Los resultados obtenidos muestran que el total de los 11 genotipos (cuadro 2) son de una aceptabilidad fenotípica buena (Escala 3). Para la determinación de esta variable se consolidó el total de las características fenotípicas del material y se determinó de manera subjetiva, con base en los objetivos de mejoramiento del presente estudio (CIAT, 1983).

Acame. El acame temprano de los tallos largos y delgados altera la distribución de las hojas, aumenta el sombrío mutuo, interrumpe el transporte de nutrientes y fotosintatos, causa esterilidad y reduce el rendimiento, además, está relacionado con otros caracteres como el diámetro del tallo y el espesor de las paredes (Jennings, 1985).

El acame causa una reducción de la calidad molinera como resultado de la fragilidad del grano, la resistencia del acame está asociada con la altura de planta, la naturaleza, extensión del sistema radicular y con el espesor y resistencia de la vaina (Poehlman, 1973).

El $82 \%$ de las líneas en estudio (cuadro 2) no presentaron acame (Escala 1); sin embargo, la línea 246 presentó plantas moderadamente volcadas en su mayoría (escala 5), y el testigo ANAR-97 mostró un 59\% con tendencia al volcamiento, lo cual indica que tuvo tendencia al acame (Escala 3).

Senescencia. La senescencia está referida a la madurez de las hojas de la planta, ya que una rápida senescencia de las hojas en el cultivo de arroz puede incurrir en detrimento del rendimiento si los granos aún no están completamente maduros (CIAT, 1983). El 100\% de los genotipos presentaron amarillamiento de las hojas superiores, ubicándolas en la categoría de senescencia intermedia (5) según la escala del CIAT (cuadro 2).

Excersión de la panícula (cm). Zeledón (1993), indica que la excersión es una variable de suma importancia para el proceso de selección de un material genético; de igual manera, destaca que una pésima excersión o la ausencia de ella, dificulta la cosecha cuando ésta se realiza de forma mecánica.

El resultado muestra que el $82 \%$ de los genotipos (cuadro 2) tuvieron categoría 1 o buena longitud de la excersión facilitando la cosecha, aunque la línea 18 y la línea 90 presentaron una categoría de Excersión moderada (Escala 3).

Longitud de la panícula (cm). Algunos estudios muestran que la longitud de las panículas oscila entre 20 y $26 \mathrm{~cm}$, cifras que fueron superadas por las 11 líneas en estudio. La longitud de panícula fue influenciada por caracteres genéticos y el ambiente, coincidiendo con Angladette (1969) y rea-
Cuadro 2. Aceptabilidad fenotípica, acame, senescencia y excersion de la panícula de 11 líneas de arroz bajo riego por inundación en Sébaco, Matagalpa. Julio -noviembre 2014

\begin{tabular}{lcccc}
\hline Líneas & $\begin{array}{c}\text { Aceptabilidad } \\
\text { fenotípica }\end{array}$ & Acame & $\begin{array}{c}\text { Escalas } \\
\text { senescencia }\end{array}$ & $\begin{array}{c}\text { Excersión de } \\
\text { panícula }\end{array}$ \\
\hline 18 & 3 & 1 & 5 & 3 \\
71 & 3 & 1 & 5 & 1 \\
72 & 3 & 1 & 5 & 1 \\
73 & 3 & 1 & 5 & 1 \\
78 & 3 & 1 & 5 & 1 \\
90 & 3 & 1 & 5 & 3 \\
109 & 3 & 1 & 5 & 1 \\
246 & 3 & 5 & 5 & 1 \\
248 & 3 & 1 & 5 & 1 \\
ANAR 97 & 3 & 3 & 5 & 1 \\
ANAR 470 & 3 & 1 & 5 & 1 \\
& & & & \\
\hline
\end{tabular}

firmando la importancia de esta variable indicada por López (1991), donde expresa que la importancia radica en que la longitud permite un mayor número de granos en la panícula.

El ANDEVA realizado mostró que hubo diferencia significativa entre las 11 líneas evaluadas y que solo 2 líneas fueron diferentes con longitud de panícula muy baja. La línea 72 y la variedad ANAR 470 presentaron la mayor longitud de panícula con $27.62 \mathrm{~cm}$ y $27.29 \mathrm{~cm}$ respectivamente siendo estadísticamente iguales. La línea 248 obtuvo el menor promedio con $22 \mathrm{~cm}$ seguido de la línea ANAR 97 con 23.32 $\mathrm{cm}$. Soto (1991) afirma que la longitud de la panícula varía entre 10 y $40 \mathrm{~cm}$ respectivamente, aunque la mayoría de las variedades comerciales están entre 20 y $24 \mathrm{~cm}$ de largo.

Altura de planta (cm). La altura del tallo principal está en función de los nudos y longitud de los entrenudos, siendo una característica varietal bien definida, aunque puede ser afectada por las condiciones ambientales (Somarriba, 1984).

La altura de planta es un factor de gran importancia agronómica al momento de la selección de una línea promisoria para su futura liberación. Mientras familias productoras de escasos recursos ubicadas en condiciones de secano medianamente favorecidos prefieren las variedades de porte alto para disminuir el efecto de las malezas. Los productores ubicados en secano favorecidos (lluvias esporádicas), toman como criterio de evaluación el uso de variedades enanas o semi enanas por la capacidad de macollamiento y menor susceptibilidad al acame (INTA, 2000).

El análisis estadístico mostró diferencia altamente significativa entre los tratamientos evaluados. El mayor rango de altura lo presentó la línea 246 con $116.33 \mathrm{~cm}$ seguido de ANAR 470 con $112.66 \mathrm{~cm}$ siendo estadísticamente iguales. La línea 109 fue la que presentó la altura más baja con $93 \mathrm{~cm}$, seguido de la línea ANAR 97 con $95.33 \mathrm{~cm}$ (cuadro 3). El CIAT (1983), expresa que la altura de la planta está influenciada por condiciones ambientales, siendo importante desde el punto de vista agronómico, por estar estrechamente relacionada con la resistencia al acame. 
Cuadro 3. Longitud de la panícula y altura de planta de 11 líneas de arroz bajo riego por inundación en Sébaco, Matagalpa. Julio -noviembre 2014

\begin{tabular}{|c|c|c|c|c|}
\hline \multirow{2}{*}{$\begin{array}{l}\begin{array}{l}\text { Líneas } \\
(\mathrm{cm})\end{array} \\
18\end{array}$} & \multicolumn{2}{|c|}{$\begin{array}{l}\text { Longitud de } \\
\text { panícula }(\mathrm{cm})\end{array}$} & \multicolumn{2}{|c|}{ Altura de Planta } \\
\hline & 25.63 & $a b c$ & 101.66 & $\mathrm{~cd}$ \\
\hline 71 & 25.38 & $a b c$ & 104.66 & bc \\
\hline 72 & 27.62 & $\mathrm{a}$ & 104.00 & $\mathrm{bc}$ \\
\hline 73 & 26.45 & $a b$ & 102.33 & $\mathrm{~cd}$ \\
\hline 78 & 26.30 & $a b$ & 99.66 & $\mathrm{~cd}$ \\
\hline 90 & 25.57 & $\mathrm{ab}$ & 98.66 & $\mathrm{~cd}$ \\
\hline 109 & 25.16 & $a b c$ & 93.33 & $\mathrm{~d}$ \\
\hline 246 & 25.77 & $a b c$ & 116.33 & $\mathrm{a}$ \\
\hline 248 & 22.00 & $\mathrm{c}$ & 103.66 & bc \\
\hline ANAR 97 & 23.32 & bc & 95.33 & $\mathrm{~cd}$ \\
\hline ANAR 470 & 27.2 & $a b$ & 112.66 & $\mathrm{ab}$ \\
\hline ANDEVA & \multicolumn{2}{|l|}{ ** } & \multicolumn{2}{|c|}{$* *$} \\
\hline P. Valor & \multicolumn{2}{|l|}{0.0023} & \multicolumn{2}{|c|}{0.001} \\
\hline C.V (\%) & \multicolumn{2}{|l|}{5.32} & \multicolumn{2}{|c|}{3.32} \\
\hline
\end{tabular}

Número de granos por panícula. Soto (1991) expresa que este componente del rendimiento, está ligado así mismo con la fertilidad o esterilidad de la panícula. Por otro lado, Tinarellí (1989) manifiesta que el número de granos por panícula está definido por su longitud, la fertilización efectuada y las condiciones térmicas donde las bajas temperaturas y lumínicas producen una elevada tasa de esterilidad.

Los resultados del análisis estadístico no mostraron diferencias significativas entre los tratamientos (Cuadro 4). El mayor promedio lo obtuvo la línea 78 con 195 granos, y la línea ANAR 97 tuvo el menor valor con 135 granos.

Fertilidad de panícula. La fertilidad de la espiguilla es un prerrequisito para obtener mayores rendimientos. El porcentaje de esterilidad normal se encuentra entre el rango de 10$15 \%$ respectivamente, aunque se puede aceptar un $20 \%$. Sin embargo, la esterilidad es común entre los genotipos de arroz en general, la cual es afectada por la temperatura, acame y esterilidad híbrida y/o genética (Jennings, 1981).

El ANDEVA reveló diferencias significativas entre los tratamientos (Cuadro 4). La línea 109 obtuvo un mayor promedio de fertilidad con 91\%; al contrario, la línea ANAR 470 presentó menor promedio con $66 \%$ de fertilidad en la panícula. Entre las líneas con mayor fertilidad están 72 con $84 \%$ y las líneas 18 y 90 con $79 \%$.

Peso de 1000 granos (g). Esta variable es expresada comúnmente al 14\% de humedad, donde del 20 al 21\% respectivamente corresponde al peso de la cáscara (Pérez y Quintani1la, 1985). López (1991) expresa que el peso de 1000 granos es un carácter estable en óptimas condiciones del cultivo y depende fundamentalmente de la variedad; sin embargo, un incremento en el rendimiento se puede lograr materiales con mayor peso, y granos largos a extra largos.

No se encontró diferencia estadística significativa respecto al peso de 1000 granos (Cuadro 4). Las líneas con mayor peso fueron ANAR 470 con $29.96 \mathrm{~g}$, línea $18 \mathrm{con}$ 29.73 g y la línea 90 con $29 . a 0 \mathrm{~g}$. ANAR 97 obtuvo el menor peso de 100 granos con $27.53 \mathrm{~g}$.
Rendimiento en granza. El rendimiento promedio nacional de arroz de riego con inundación es de $2,757.04 \mathrm{~kg} / \mathrm{ha}$ (MAGFOR, 2006). Angladette (1969), resalta que este carácter está determinado por el genotipo, la ecología del lugar y el manejo agronómico. El ANDEVA realizado para esta variable no se encontró diferencia significativa entre los 11 genotipos evaluados (cuadro 4). Las líneas 72 y 90 obtuvieron el mayor rendimiento con $6818.18 \mathrm{~kg} / \mathrm{ha}$ al contrario las líneas 78,246 y 73 obtuvieron $5757.58 \mathrm{~kg} / \mathrm{ha}, 5909.09 \mathrm{~kg} / \mathrm{ha} \mathrm{y}$ $5969.7 \mathrm{~kg} /$ ha respectivamente.

Cuadro 4. Número de granos por panícula, fertilidad de los granos de la panícula, peso de mil granos en granza 9 líneas de arroz y dos testigos comerciales en el Valle de Sébaco, 2014

\begin{tabular}{|c|c|c|c|c|}
\hline Líneas & $\begin{array}{l}\text { Número } \\
\text { de granos } \\
\text { por panícula }\end{array}$ & $\begin{array}{l}\% \text { de fertilidad } \\
\text { de los granos }\end{array}$ & $\begin{array}{l}\text { Peso de mil } \\
\text { granos }(\mathrm{g})\end{array}$ & $\begin{array}{l}\text { Rendimiento } \\
\text { arroz granza } \\
(\mathrm{kg} / \mathrm{ha})\end{array}$ \\
\hline 18 & 174 & $a b$ & 29.73 & 6363.64 \\
\hline 71 & 173 & 67 & 28.46 & 6121.21 \\
\hline 72 & 175 & 84 & 28.73 & 6818.18 \\
\hline 73 & 165 & 71 & 28.86 & 5969.70 \\
\hline 78 & 195 & 70 & 28.76 & 5757.58 \\
\hline 90 & 169 & 79 & 29.10 & 6818.18 \\
\hline 109 & 138 & 91 & 28.13 & 6757.58 \\
\hline 246 & 176 & 72 & 28.43 & 5909.09 \\
\hline 248 & 157 & 71 & 28.10 & 6515.15 \\
\hline ANAR 97 & 135 & 72 & 27.53 & 6060.61 \\
\hline ANAR 470 & 184 & 66 & 29.96 & 6515.15 \\
\hline ANDEVA & NS & ** & NS & NS \\
\hline P. Valor & 0.0516 & 0.0028 & 0.083 & 0.4109 \\
\hline C.V $(\%)$ & 12.33 & 8.75 & 2.99 & 10.06 \\
\hline
\end{tabular}

Calidad industrial. Después del rendimiento, la calidad industrial del grano es la característica más importante considerada por los fitomejoradores, debido a que los consumidores generalmente aceptan una variedad por el sabor, textura, aroma y aspecto (De Datta, 1986).

La calidad industrial es el resultado de la relación de numerosos factores físicos del grano como tamaño, forma, peso de la cascarilla, pigmentación, dureza, temperatura de gelatinización y contenido de amilosa. Otros factores a tomar en cuenta son el manejo de la cosecha, recolección, secado, transporte, procesamiento y almacenamiento (CIAT, 1986).

Para determinar esta variable se tomaron $200 \mathrm{~g}$ de arroz en granza de las 11 líneas y las 2 variedades, representando el $100 \%$ del peso total de la muestra. Respecto al rendimiento de pilada, grano entero, relación grano entero: quebrado, la línea 248 obtuvo los mayores promedios con $70.33 \%, 61.33 \%$ y $89 \%$ respectivamente. Las variedades testigo presentaron bajos resultados en estas variables (cuadro 5) presentando la variedad ANAR 97 los valores más bajos en todas las pruebas de calidad molinera con $68.33 \%$, $37.66 \%$ y $60 \% \%$, respectivamente. Cuando se continúe con los ensayos regionales se tomarán en cuenta los parámetros de arroz integral, arroz oro y el porcentaje de granza después del trillado y pulido, así como la calidad molinera arroz entero y arroz quebrado. 
Cuadro 5. Análisis de la calidad industrial de 11 cultivares de arroz bajo condiciones de riego evaluados en Sebaco, Matagalpa. Julio -noviembre 2014

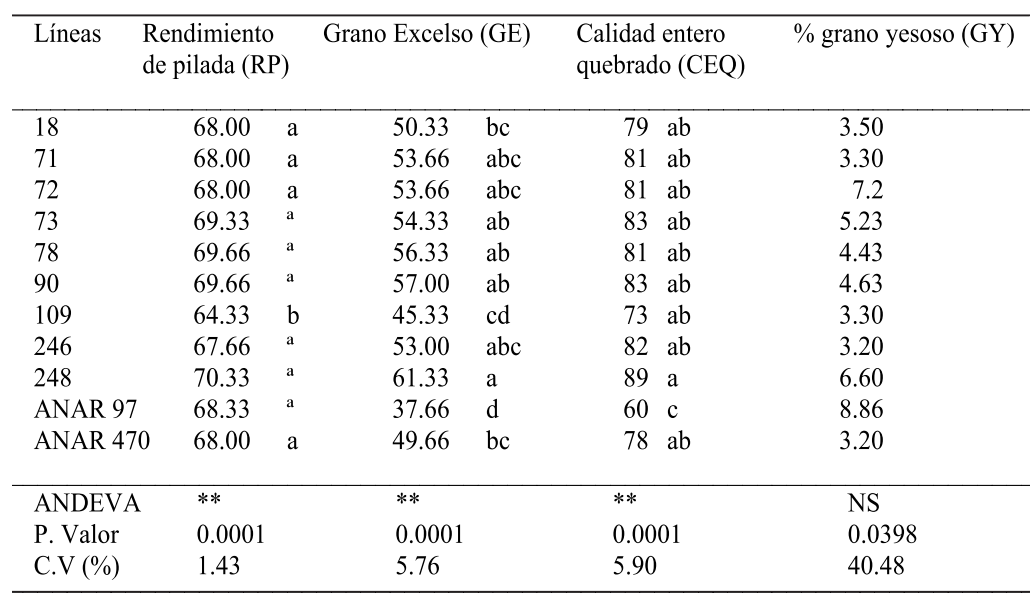

\section{CONCLUSIONES}

La línea 246 y la variedad ANAR 97 presentaron cierto índice de acame y las líneas 18 y 20 presentaron una moderada longitud de excersión. Las demás líneas no presentaron acame y buena longitud de la excersión.

De los 9 genotipos evaluados, 6 son altamente productivos superando a las variedades ANAR 97 y ANAR 470 oscilando sus rendimientos entre $6121 \mathrm{~kg} / \mathrm{ha}$ y $6818 \mathrm{~kg} / \mathrm{ha}$ siendo candidatos a pruebas regionales.

La línea 248 presentó los mejores valores de rendimiento de arroz oro, mayor porcentaje de grano entero y mejor relación grano entero grano quebrado.

\section{REFERENCIAS BIBLIOGRÁFICAS}

Angladette, A. 1969. El arroz. Colección agricultura tropical. Editorial BLUME. Barcelona, ES. 867 p.

Balladares, E; Espinoza, N. 1995. Pruebas observacionales de rendimiento de doce líneas y cuatro variedades de arroz, UNA, Managua, Nicaragua, 32 p.

Cardoza, J; González, E. 2004. Evaluación y pruebas de rendimientos de catorce líneas promisorias y dos variedades comerciales de arroz (Oryza sativa L.) bajo condiciones de riego en el Valle de Sébaco, Matagalpa. Primera 2003. Tesis Ingeniero Agrónomo. Universidad Nacional Agraria (UNA). Managua, Nicaragua. 35 p.

CIAT (Centro Internacional de Agricultura Tropical). 1986. Arroz: Investigación y producción. Cali, CO. 696 p. 1985. Arroz: investigación y producción, Cali, CO. 653 p. 1983. Componentes del rendimiento. Auxiliar didáctico. No 001. Cali, CO. 15 p. 1981. Crecimiento y etapas de desarrollo de la planta de arroz. Cali, CO. 36 p.

Contín, A. 1990. Manual de producción de arroz. 2 ed. Limusa. México D.F, MX. 426 p.

De Datta, SK. 1986. Producción de arroz: fundamento y práctica. Limusa, México DF. MX. 690 p.

Herrera, B. 2008. Departamento de Suelos. D.G.E.T-MAGFOR. Managua, Nicaragua.

Holdrige, LR. 1982. Ecología con base en zonas de vida. IICA. San José, CR. 216 p.

INTA (Instituto Nicaragüense de Tecnología Agropecuaria). 2000. Validación de genotipos mejorados de arroz en ambientes húmedos, Masatepe, Nicaragua. 5 p.

1996. Guía tecnológica del cultivo de arroz, Managua, NI. 21 p.

INETER (Instituto Nicaragüense de Estudios Territoriales). 2015. Boletín informativo de las condiciones climáticas de Darío, Matagalpa, 2014. Estación meteorológica del Valle de Sébaco, San Isidro, Matagalpa, NI. 5 p.

Jennings, PR. 1981. Mejoramiento de arroz. 237 p.

1985. Mejoramiento del arroz: investigación y producción. Editorial CIAT. Palmira, Colombia. 231 p.

López, BL. 1991. Cultivos herbáceos: Cereales. 91 p.

MAGFOR (Ministerio Agropecuario y Forestal, NI). 2002. Informe anual de producción agropecuaria - Ciclo Agrícola 2002/2003 y período pecuario 2002. Dirección de Estadísticas. Managua, NI. 86 p.

Munguía, R; Obregón, J. 2000. Prueba avanzada de rendimiento de 11 líneas promisorias de arroz para condiciones de secano, Rivas, Nicaragua. 75 p.

Pérez, JW; Acevedo, A; Quintanilla. 1985. Relación entre el rendimiento y carácter morfológico en arroz en Nicaragua. Ciencia y Tecnología en la Agricultura. La Habana, CU. 230 p.

SAS INSTITUTE, INC. 2002. SAS Introductory guide for personal computers: Statistics. Versión 9.1. SAS institute, Inc. Cary, North Calorina, US. $111 \mathrm{p}$.

Somarriba, C. 1984. Cultivo del arroz. Universidad Nacional Agraria (UNA), Managua, NI. 35 p.

Soto, B. 1991. Estudio de observación de 20 variedades USA y siete líneas promisorias nacionales en comparación con dos testigos comerciales de arroz, Managua, Nicaragua.

Tinarelli, A. 1989. El arroz. 2 ed. EDAGRICOLE. Bologna, IT. 298 p.

Zeledón, P. 1993. Estudio de observación de 112 líneas de arroz (Oryza sativa L.) VIOAL- 92 bajo el ecosistema de riego Managua, Nicaragua. 29 p. 